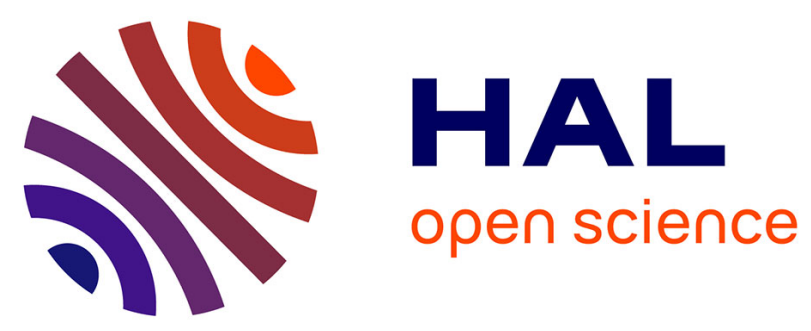

\title{
Pinball liquid phase from Hund's coupling in frustrated transition-metal oxides
}

\author{
Arnaud Ralko, Jaime Merino, Simone Fratini
}

\section{To cite this version:}

Arnaud Ralko, Jaime Merino, Simone Fratini. Pinball liquid phase from Hund's coupling in frustrated transition-metal oxides. Physical Review B: Condensed Matter and Materials Physics (1998-2015), 2015, 91 (16), pp.165139. 10.1103/PhysRevB.91.165139 . hal-01219131

\section{HAL Id: hal-01219131 \\ https://hal.science/hal-01219131}

Submitted on 1 Feb 2019

HAL is a multi-disciplinary open access archive for the deposit and dissemination of scientific research documents, whether they are published or not. The documents may come from teaching and research institutions in France or abroad, or from public or private research centers.
L'archive ouverte pluridisciplinaire HAL, est destinée au dépôt et à la diffusion de documents scientifiques de niveau recherche, publiés ou non, émanant des établissements d'enseignement et de recherche français ou étrangers, des laboratoires publics ou privés. 


\title{
Pinball liquid phase from Hund's coupling in frustrated transition-metal oxides
}

\author{
Arnaud Ralko, ${ }^{1,2}$ Jaime Merino, ${ }^{3}$ and Simone Fratini ${ }^{1,2}$ \\ ${ }^{1}$ Institut NEEL, Université Grenoble Alpes, F-38042 Grenoble, France \\ ${ }^{2}$ Institut NEEL, CNRS, F-38042 Grenoble, France \\ ${ }^{3}$ Departamento de Física Teórica de la Materia Condensada, Condensed Matter Physics Center (IFIMAC) \\ and Instituto Nicolás Cabrera, Universidad Autónoma de Madrid, Madrid 28049, Spain
}

(Received 15 December 2014; revised manuscript received 16 April 2015; published 30 April 2015)

\begin{abstract}
The interplay of nonlocal Coulomb repulsion and Hund's coupling in the $d$-orbital manifold in frustrated triangular lattices is analyzed by a multiband extended Hubbard model. We find a rich phase diagram with several competing phases, including a robust pinball liquid phase, which is an unconventional metal characterized by threefold charge order, bad metallic behavior, and the emergence of high-spin local moments. Our results naturally explain the anomalous charge-ordered metallic state observed in the triangular layered compound $\mathrm{AgNiO}_{2}$. The potential relevance to other triangular transition-metal oxides is discussed.
\end{abstract}

DOI: 10.1103/PhysRevB.91.165139

\section{INTRODUCTION}

Materials with competing electronic interactions on triangular lattices are a fertile ground for novel phenomena and original quantum phases, such as the spin-liquid behavior [1] observed in organic $\left(\kappa-(\mathrm{BEDT}-\mathrm{TTF})_{2} \mathrm{Cu}_{2}(\mathrm{CN})_{3}\right.$, $\left.\mathrm{Me}_{3} \mathrm{EtSb}\left[\mathrm{Pd}(\mathrm{dmit})_{2}\right]_{2}\right)$ and inorganic $\left(\mathrm{Cs}_{2} \mathrm{CuCl}_{4}\right)$ quasi-twodimensional materials. Geometrical frustration can play a similar role in charge-ordering phenomena, leading to puzzling unconventional metallic and superconducting states. Remarkable examples are found in the quarter-filled organic salts $\theta$-(BEDT-TTF $)_{2} X[2,3]$, the layered cobaltates $\mathrm{Na}_{x} \mathrm{CoO}_{2}$ [4-7], and the transition-metal dichalcogenide $1 T-\mathrm{TaS}_{2}$ [8].

An interesting yet less explored member of this category is $\mathrm{AgNiO}_{2}$, a layered oxide compound with a triangular planar lattice structure, whose properties reflect a rich interplay between magnetic, orbital, and charge degrees of freedom. This system presents a robust threefold chargeordered phase, which is stable above room temperature $\left(T_{\mathrm{CO}}=\right.$ $365 \mathrm{~K})$ and undergoes magnetic ordering only at much lower temperatures, $T_{N}=20 \mathrm{~K}$ [9-11]. Contrary to the common behavior observed in oxides with Jahn-Teller active ions, the charge ordering in this material is not associated with any structural distortion, indicative of a purely electronic driving mechanism. Furthermore, the ordering is partially frustrated by the triangular lattice geometry, causing the electronic system to spontaneously separate into localized magnetic moments, residing on a superlattice of charge-rich $\mathrm{Ni}$ sites, and itinerant electrons moving on the honeycomb lattice formed by the remaining charge-poor Ni sites. The material is therefore metallic throughout the charge-ordered phase, which contrasts with the situation in nonfrustrated perovskite nickelates [12,13], $R \mathrm{NiO}_{3}$, where charge order invariably leads to an insulating behavior. The high values of the electrical resistivity and its anomalous temperature dependence in $\mathrm{AgNiO}_{2}$ [14-17], however, indicate bad metallic behavior, also supported by the observation of a large pseudogap in photoemission experiments [18], an anomalous Seebeck coefficient [14,15], and a large specific-heat coefficient [19].

In this work, we analyze a multiband microscopic model which takes explicit account of electronic correlations to demonstrate that the emergence of charge-ordered phases with unconventional metallic properties is a natural outcome
PACS number(s): 71.10.Hf, 71.30.+h, 73.20.Qt, 74.70.Kn

in frustrated triangular oxides with both strong Coulomb interactions and Hund's coupling away from half-filling. Our results show that the combination of on-site and off-site Coulomb repulsion and Hund exchange stabilizes a robust pinball liquid phase [20-25], a quantum phase where the electrons spontaneously separate into coexisting localized "pins" exhibiting Mott physics and itinerant "balls" moving on the remaining honeycomb lattice. We argue that the chargeordered metallic phase of $\mathrm{AgNiO}_{2}$ is a neat experimental realization of such a pinball liquid, which explains many experimental features such as the threefold ordering pattern with strong charge disproportionation, the presence of large local moments, and the "bad" metallic behavior.

\section{TWO-ORBITAL MICROSCOPIC DESCRIPTION}

In $\mathrm{AgNiO}_{2}$ the $d$ orbitals of $\mathrm{Ni}\left(t_{2 g}^{6} e^{1}{ }_{g}\right.$ configuration, formal valence $\mathrm{Ni}^{3+}$ ) split into an upper $e_{g}$ doublet occupied by one electron and a completely filled lower $t_{2 g}$ triplet that are separated by a crystal-field gap of $\sim 2 \mathrm{eV}[17,18,26]$. Labeling by $\tau=1,2$ the $e_{g}$ orbitals $d_{3 z^{2}-r^{2}}$ and $d_{x^{2}-y^{2}}$ and neglecting the low-lying $t_{2 g}$ orbitals, we write the following two-orbital extended Hubbard model:

$$
H=-t \sum_{\langle i j\rangle} \sum_{\tau, \sigma}\left(d_{i, \tau \sigma}^{\dagger} d_{j, \tau \sigma}+\text { H.c. }\right)+H_{\text {Hund }}+H_{\mathrm{V}} .
$$

The first term describes $e_{g}$ electrons moving on the triangular lattice of $\mathrm{Ni}$ ions with transfer integrals $t$ at a density $n=$ 1 , which nominally corresponds to one-quarter filling (one electron per two orbitals per site). These electrons interact on each atomic site via the Hund coupling, as described by the standard Kanamori Hamiltonian [27,28]:

$$
\begin{aligned}
H_{\text {Hund }}= & U \sum_{i, \tau} n_{i \tau \uparrow} n_{i \tau \downarrow}+\left(U-2 J_{H}\right) \sum_{i, \tau \neq \tau^{\prime}} n_{i \tau \uparrow} n_{i \tau^{\prime} \downarrow} \\
& +\left(U-3 J_{H}\right) \sum_{i, \tau<\tau^{\prime}, \sigma} n_{i \tau \sigma} n_{i \tau^{\prime} \sigma} \\
& -J_{H} \sum_{i, \tau \neq \tau^{\prime}}\left(d_{i \tau \uparrow}^{+} d_{i \tau \downarrow} d_{i \tau^{\prime} \downarrow}^{+} d_{i \tau^{\prime} \uparrow}-d_{i \tau \uparrow}^{+} d_{i \tau \downarrow}^{+} d_{i \tau^{\prime} \downarrow} d_{i \tau^{\prime} \uparrow}\right) .
\end{aligned}
$$




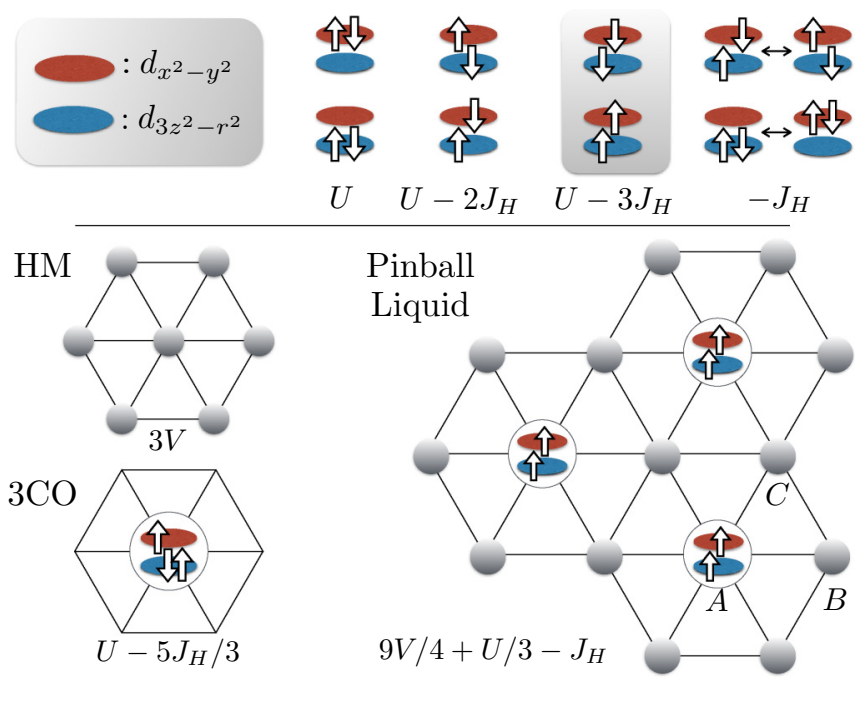

FIG. 1. (Color online) (top) Atomic processes described by the Hund interaction $H_{\text {Hund }}$, with the corresponding coupling constants, highlighting the high-spin configuration of lowest energy; the disks of different colors represent the orbitals $d_{3 z^{2}-r^{2}}$ and $d_{x^{2}-y^{2}}$. (bottom) Electronic configurations in the homogeneous-metal (HM), threefold charge-ordered (3CO), and pinball liquid phases on the triangular lattice, with the corresponding electrostatic energies. Arrows represent localized moments (pins); gray disks are the itinerant electrons (balls).

This comprises intraorbital and interorbital repulsion as well as pair hopping and spin-flip processes, as illustrated in Fig. 1. We also consider a nearest-neighbor Coulomb repulsion term $H_{\mathrm{V}}=V \sum_{\langle i j\rangle} n_{i} n_{j}$ as the driving force for charge disproportionation, where $n_{i}=\sum_{\tau, \sigma} n_{i, \tau \sigma}$ is the total density operator at site $i$, with $n_{i, \tau \sigma}=d_{i, \tau \sigma}^{+} d_{i, \tau \sigma}$.

The competition between the different terms in the Hamiltonian (1) can be understood from the following electrostatic considerations. For sufficiently weak interactions, the system is a homogeneous metal (HM) with $n_{i}=n=1$. Because the interaction energy $E_{\mathrm{HM}}=3 V$ of this uniform configuration increases with $V$, a charge-ordered configuration will be preferred at large $V$ in order to minimize the electrostatic energy cost. On the triangular lattice, this is achieved by ordering electrons on a three-sublattice structure (sublattices $A, B, C$ ) with $n_{A}=3$ electrons per site on one sublattice and all other sites empty. The interaction energy of this threefold charge order (3CO), sketched in Fig. 1, is purely atomic, $E_{3 \mathrm{CO}}=U-5 J_{H} / 3$ per site. It has no energy cost associated with the off-site Coulomb repulsion and is therefore favored at large $V$.

From previous studies of the extended Hubbard model in the single-band case [23-25] it is known that an intermediate phase can be stabilized between the $3 \mathrm{CO}$ and the homogeneous metal. In this phase, termed pinball liquid (PL), part of the electron density of the charge-rich sites (pins) spills out to the neighboring unoccupied sites (balls) in order to reach a favorable compromise between local and nonlocal Coulomb interactions. The additional microscopic processes included in the present multiband case, which favor high-spin configurations, play a key role in this scenario: the maximum
Hund's exchange energy is achieved in ions with a total spin-1 configuration, where precisely two electron spins are aligned (Fig. 1). Therefore, a phase which maximizes the number of doubly occupied sites will be naturally promoted for sufficiently large $J_{H}$, stabilizing a pinball liquid state with $n_{A}=2$ on the charge-rich sites instead of $n_{A}=3$.

The key role of $J_{H}$ in stabilizing the PL can be assessed quantitatively by comparing its energy, $E_{\mathrm{PL}}=9 \mathrm{~V} / 4+U / 3-$ $J_{H}$, with that of the $3 \mathrm{CO}$ and $\mathrm{HM}$ calculated previously. The PL is favored when $U_{c}^{(1)}<U<U_{c}^{(2)}$, with $U_{c}^{(1)}=J_{H}+$ $(27 / 8) V+C_{1}$ and $U_{c}^{(2)}=3 J_{H}+(9 / 4) V+C_{2}$ (the constant terms arise from the kinetic-energy gain of mobile electrons in the PL and HM phases, which both scale proportionally to $t$ [21]). This energetic argument predicts that (i) the PL phase emerges above a critical value of $J_{H} / U$ and (ii) its area spreads upon increasing $J_{H} / U$ and diverges for $J_{H} / U=1 / 3$.

\section{PHASE DIAGRAM}

We solve Eq. (1) by employing two complementary methods. We first apply unrestricted Hartree-Fock (UHF) meanfield theory allowing for solutions breaking any symmetry, which guides us systematically through the whole phase diagram. To address the effects of electron correlations that were neglected in previous theoretical treatments $[9,12,29]$ and that we demonstrate here to be crucial in the region of experimental relevance, we then use single-site dynamical mean-field theory (DMFT). This is expected to be particularly accurate in systems with geometrical frustration or with large coordination, where the spatial range of nonlocal correlations is suppressed. We focus on solutions with threefold translational symmetry breaking, restricting ourselves to paramagnetic phases and ignoring the possible ordering on the minority sublattice, which leaves us with two two-orbital impurity models describing the charge-rich and charge-poor sites coupled only through the hopping. Note that in the DMFT, the on-site correlations are treated exactly, while a Hartree decoupling is employed for the nearest-neighbor interaction $V$. The full DMFT self-consistent loop is evaluated using Lanczos diagonalization until self-consistency. For technical details see Refs. [25,30].

We report in the main panel of Fig. 2 the results obtained by varying the ratio $J_{H} / U$ in the interval $(0.05-0.3)$ for an experimentally relevant value of the intersite Coulomb repulsion $V / t=2$ [31]. As expected from the electrostatic argument above, a prominent PL phase emerges in a broad region of the phase diagram between the homogeneous metal and the $3 \mathrm{CO}$. The boundaries of the PL region, determined by the conditions $n_{A}=2$ (onset of PL) and $n_{A}=1(\mathrm{HM})$, are shown as points (gray for UHF, black for DMFT) and closely follow the analytical predictions $U_{c}^{(1)}$ and $U_{c}^{(2)}$, drawn as dotted lines (here adjusted by setting the constants $C_{2}=-C_{1}=0.8 t$ ). The area covered by the PL spreads upon increasing the Hund coupling and attains values of $U$ that are quite typical for transition-metal oxides. This should be contrasted with the case where the Hund coupling is small or absent, in which case the local Coulomb repulsion prevents any possibility of charge ordering and a homogeneous metal is stabilized instead (left side of Fig. 2). 


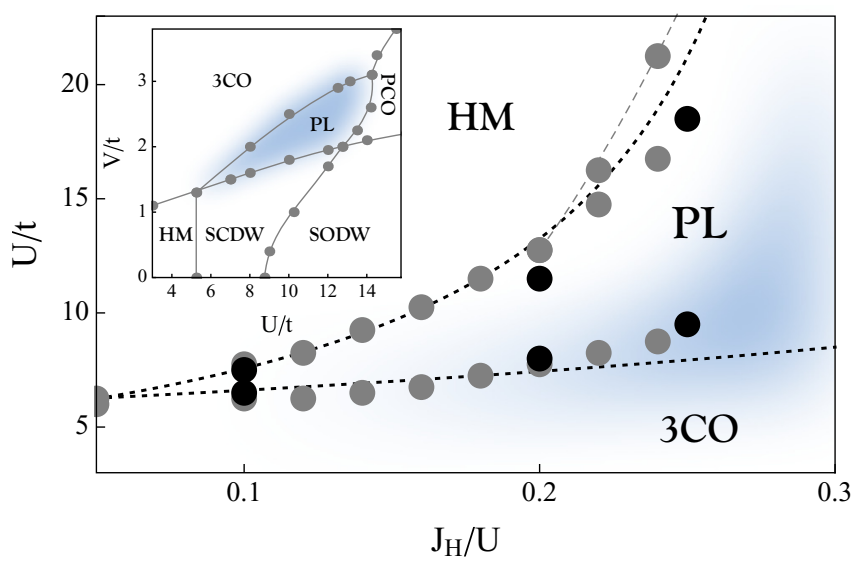

FIG. 2. (Color online) Phase diagram of the two-orbital extended Hubbard model on the triangular lattice obtained from DMFT (black points) and UHF (gray points) for a representative value $V / t=2$. The dotted lines are the phase boundaries $U_{c}^{(1)}$ and $U_{c}^{(2)}$ given in the text. The dashed line indicates charge order within the charge-poor sublattice as found in the UHF solution. The inset shows the UHF phase diagram in the $(U, V)$ plane for $J_{H} / U=0.2$.

The physics of this model is even richer if we allow for more general broken-symmetry states, as presented in the inset of Fig. 2 for a representative value $J_{H} / U=0.2$. The metal at low $V$ has further symmetry breaking for sufficiently large $U$, corresponding to spin/charge density waves (SCDW) and spin/orbital density waves (SODW). An additional transition also appears within the pinball phase at large $U$ and $V$, corresponding to the ordering of the mobile electrons on the honeycomb lattice (pinball charge order, PCO). These results will be discussed elsewhere [32].

\section{PINBALL LIQUID}

To further characterize the PL phase, we show in Fig. 3 several physical properties obtained by DMFT at different values of the $J_{H} / U$ ratio. The key quantity that controls the behavior of the system is the average electron density in the different sublattices, shown in Fig. 3(a). Starting from the $3 \mathrm{CO}$ phase, the charge-rich sublattice density is progressively reduced with $U$ until it reaches $n_{A}=2$. The onset of the PL is signaled by a kink at this point, followed by a plateau which develops at large $J_{H} / U$ extending all the way up to the HM phase. To demonstrate that such a "lock-in" of the density is closely related to the existence of a high-spin configuration on the pins, in Fig. 3(b) we show the value of the local magnetic moment evaluated for the same values of the microscopic parameters. Closely following the behavior of the density, a plateau is observed in the magnetic moment too, with a maximum in correspondence of $n_{A}=2$, as expected (arrows). Interestingly, upon increasing $J_{H}$ the fluctuating magnetic moment takes large values approaching the ideal limit $S_{A}=1$ $\left(S_{A} \approx 0.85\right.$ at $U=9 t$ and $\left.J_{H} / U=0.25\right)$, indicative of strong local correlations.

The evolution of the quasiparticle weight, shown in Fig. 3(c), reveals how in the presence of a sizable $J_{H}$, a large mass enhancement, $m^{*} / m_{b}=1 / Z$, occurs on the pins already for moderate values of $U \lesssim W$ (here $W=9 t$ is the
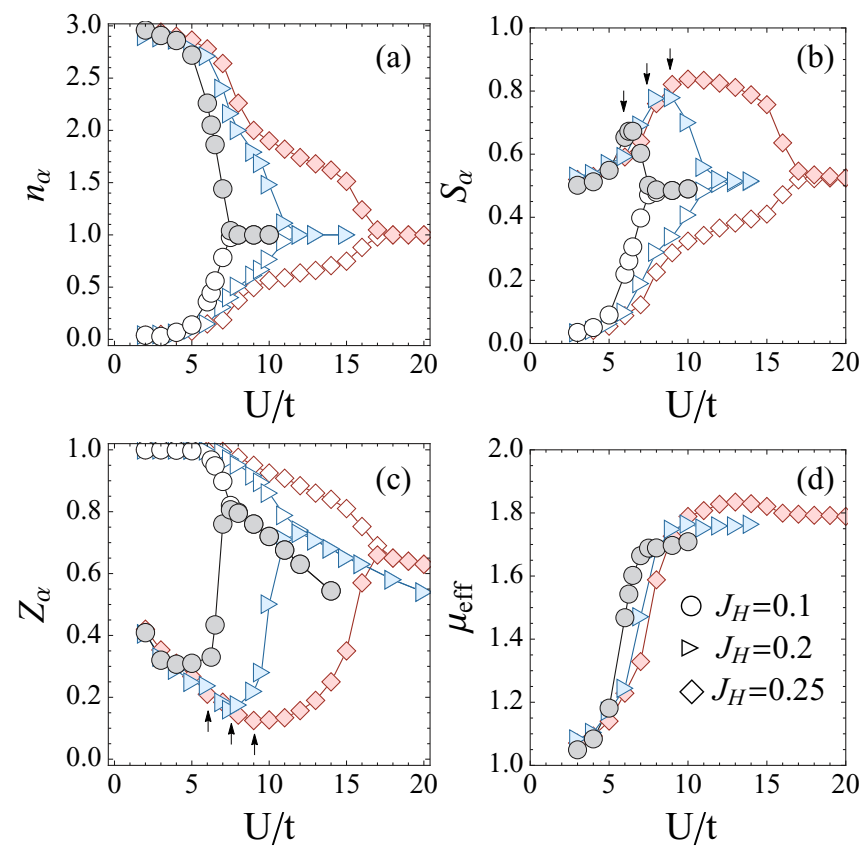

FIG. 3. (Color online) (a) Electronic density $n_{\alpha}$, (b) magnetic moment $S_{\alpha}$ obtained from $\left\langle S_{\alpha}^{2}\right\rangle=S_{\alpha}\left(S_{\alpha}+1\right)$, and (c) quasiparticle renormalization $Z_{\alpha}$ on the charge-rich ( $\alpha=A$; solid symbols) and charge-poor ( $\alpha=B, C$; open symbols) sublattices for $V / t=2$ and $J_{H} / U=0.1$ (circles), 0.2 (triangles), and 0.25 (diamonds). (d) Total effective Bohr magneton (see text).

bandwidth on the triangular lattice). This happens because the density on the charge-rich sublattice is locked around half filling (two electrons in two orbitals), which corresponds to the maximally correlated case in the presence of Hund's coupling [33]. Accordingly, the minimum of $Z_{A}$ coincides with the value where $n_{A}=2$, indicated by arrows. At the same time, the mass of the minority electrons remains close to the band value, owing to their low concentration in the honeycomb lattice. Figure 3(c) also shows that the mass renormalization of the majority electrons in the PL phase at intermediate $U$ is much stronger than that of the homogeneous metallic phase at large $U$. Upon reaching the HM phase, the quasiparticle weight jumps back to a less correlated value. It then gradually decreases with $U$ towards the Mott transition expected at a value $U / t \sim 36$ [34]. Note that within the HM phase the quasiparticle weight at a given $U$ is found to increase with $J_{H}$, as expected for a two-orbital system with one electron per site [34].

\section{DISCUSSION}

We now analyze the consequences of the present theoretical scenario, in connection with existing experiments on $\mathrm{AgNiO}_{2}$. To make a quantitative comparison with the measured Curie-Weiss susceptibility [10,16,17], we report in Fig. 3(d) the effective Bohr magneton, $\mu_{\text {eff }}=g \mu_{B} \sqrt{\left\langle S_{\text {eff }}^{2}\right\rangle}$, as obtained from the effective moment per site evaluated in DMFT: $\left\langle S_{\text {eff }}^{2}\right\rangle \approx \frac{\left\langle S_{A}^{2}\right\rangle+\left\langle S_{B}^{2}\right\rangle+\left\langle S_{C}^{2}\right\rangle}{3}$. We see that values much larger than the density-functional-theory-localdensity-approximation estimates, $\mu_{\mathrm{eff}}=1.3-1.5$ [9], and 

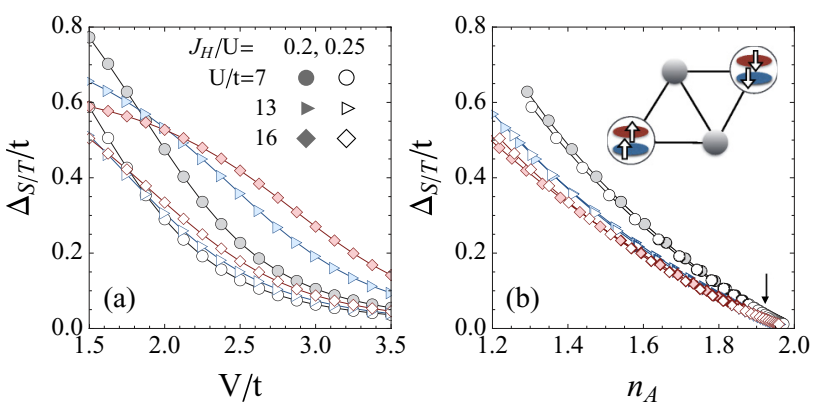

FIG. 4. (Color online) (a) Singlet-triplet gap for the model (1) on a four-site cluster, in units of $t$. (b) Same as in (a), plotted vs the charge-rich sublattice density.

quantitatively consistent with the experimental range of results, $\mu_{\mathrm{eff}}=1.81-1.96$, are naturally reached in the presence of substantial electronic correlations in the PL and remain high also in the HM phase at larger $U$.

Second, we discuss the origin of the magnetic ordering observed at low temperatures $[9,10,35]$. To assess the magnitude of the antiferromagnetic (AF) coupling $J$ between nearest-neighboring local moments in the PL mediated by virtual superexchange processes via the charge-poor sites, we diagonalize Eq. (1) on a minimal four-site cluster with open boundary conditions [illustrated in the inset of Fig. 4(b)] and calculate the singlet-triplet gap $\Delta_{S / T}$ in the excitation spectrum, which coincides with $J$ in the Heisenberg limit [36]. Figure 4(a) shows that $J$ strongly decreases with $V$ and, to a lesser extent, also with $U$. To highlight the role played by the electron density on the pins, we redraw the results as a function of $n_{A}$ in Fig. 4(b). The data collapse in a narrow region, which we use to estimate $n_{A}$ from the experimental value of $J$. From the magnetic-ordering temperature, $T_{N}=20 \mathrm{~K}$ and using $T_{N} \simeq 0.3 \mathrm{~J}$ based on the classical Monte Carlo simulations of Ref. [37], we obtain $J \simeq 6 \mathrm{meV}=0.03 t$ for $t=0.2 \mathrm{eV}$. Such a low value of the magnetic-ordering temperature implies that the system is very close to the integer filling $n_{A}=2$ (we estimate $n_{A} \gtrsim 1.9$, indicated by an arrow), locating $\mathrm{AgNiO}_{2}$ in the strongly correlated pinball phase.

$\mathrm{X}$-ray and neutron scattering experiments do indicate substantial threefold charge disproportionation [10,11,38], compatible with the emergence of large magnetic moments on the charge-rich sites $[9,10]$. In our scenario, an AF coupling between itinerant and localized species [25] leads to the screening of the pin local moments giving way to Fermiliquid behavior at low temperatures as in heavy fermions. Above the coherent-incoherent crossover temperature $T^{*}$, quasiparticles are destroyed due to the scattering of the itinerant carriers by the unscreened pin moments, which has several experimental manifestations. The measured resistivity indeed displays typical Fermi-liquid behavior $\rho \sim T^{2}$ above the Néel temperature $T_{N}=20 \mathrm{~K}$, albeit with anomalously large absolute values $(\gtrsim 1 \mathrm{~m} \Omega \mathrm{cm})$ [14-17], which crosses over to a (sub)linear $T$ dependence [16] at temperatures above $T^{*} \simeq 150 \mathrm{~K}$. At lower temperatures, the resistivity undergoes a sharp drop below $T_{N}$ which has been associated $[10,19]$ with the suppression of such scattering. The Seebeck coefficient increases linearly with temperature up to about $100 \mathrm{~K}$, as expected in a metal, but then it reaches a maximum around $T^{*}$ and changes sign at $260 \mathrm{~K}[14,15]$. A crossover in the Curie-Weiss susceptibility is also observed close to $T^{*}$ [17]. Finally, the value of the specific-heat coefficient, $\gamma=C_{v} / T$, within the AF phase suggests an appreciable mass enhancement, $m^{*} / m_{b}=2.6$ [19], intermediate between the values calculated for pins and balls in Fig. 3(c).

\section{OUTLOOK}

Previous works describing orbitally degenerate transitionmetal oxides with quarter-filled bands have focused on models where frustration plays a minor role, leading to charge-ordered insulating states $[12,13]$. Here, we have demonstrated that Coulomb-induced charge ordering on frustrated triangular lattices leads to the emergence of a robust metallic pinball liquid phase stabilized by Hund's coupling acting on the $d$-orbital manifold. Such a phase presents characteristics qualitatively similar to heavy fermions and bad metallic behavior associated with the Kondo coupling between localized moments and itinerant carriers, consistent with what is observed in the charge-ordered metal $\mathrm{AgNiO}_{2}$. Optical conductivity experiments in this material could be used to observe the concomitant destruction of the Drude peak [39-41] above the coherentincoherent crossover scale $T^{*}$. Applying external pressure may destroy the magnetic order at a quantum critical point, giving way to a Fermi-liquid state, as observed in heavy-fermion materials [42] and the nickel oxypnictide CeNiAsO [43]. Other phases found here such as the spin/orbital stripe states or the PCO phase with ordering of the ball sites could also be realized, as is the case in adsorbates deposited on metal surfaces [30]. Finally, a similar interplay of multiorbital physics, electronic correlations, and charge ordering may occur on other triangular compounds such as $\mathrm{Ag}_{2} \mathrm{NiO}_{2}$ [44], the $\mathrm{Ba}_{3} \mathrm{~B}^{\prime} \mathrm{Ru}_{2} \mathrm{O}_{9}$ ruthenates [45], and the superconducting cobaltates [4-7], as well as other geometrically frustrated lattices.

\section{ACKNOWLEDGMENTS}

Discussions with C. Février are gratefully acknowledged. J.M. acknowledges financial support from MINECO (MAT2012-37263-C02-01). This work is supported by the French National Research Agency through Grants No. ANR-12-JS04-0003-01 SUBRISSYME and No. ANR-2010BLANC-0406-0 NQPTP.
[1] L. Balents, Nature (London) 464, 199 (2010).

[2] C. Hotta, Crystals 2, 1155 (2012).

[3] F. Kagawa, T. Sato, K. Miyagawa, K. Kanoda, Y. Tokura, K. Kobayashi, R. Kumai, and Y. Murakami, Nat. Phys. 9, 419 (2013).
[4] K. Takada, H. Sakurai, E. Takayama-Muromachi, F. Izumi, R. A. Dilanian, and T. Sasaki, Nature (London) 422, 53 (2003).

[5] N. P. Ong and R. J. Cava, Science 305, 52 (2004).

[6] M.-H. Julien, C. de Vaulx, H. Mayaffre, C. Berthier, M. Horvati, V. Simonet, J. Wooldridge, G. Balakrishnan, M. R. Lees, D. P. 
Chen, C. T. Lin, and P. Lejay, Phys. Rev. Lett. 100, 096405 (2008).

[7] H. Alloul, I. R. Mukhamedshin, A. V. Dooglav, Ya. V. Dmitriev, V.-C. Ciomaga, L. Pinsard-Gaudart, and G. Collin, Phys. Rev. B 85, 134433 (2012).

[8] B. Sipos, A. F. Kusmartseva, A. Akrap, H. Berger, L. Forró, and E. Tutis, Nat. Mater. 7, 960 (2008).

[9] E. Wawrzyńska, R. Coldea, E. M. Wheeler, I. I. Mazin, M. D. Johannes, T. Sörgel, M. Jansen, R. M. Ibberson, and P. G. Radaelli, Phys. Rev. Lett. 99, 157204 (2007).

[10] E. Wawrzyńska, R. Coldea, E. M. Wheeler, T. Sörgel, M. Jansen, R. M. Ibberson, P. G. Radaelli, and M. M. Koza, Phys. Rev. B 77, 094439 (2008).

[11] G. L. Pascut, R. Coldea, P. G. Radaelli, A. Bombardi, G. Beutier, I. I. Mazin, M. D. Johannes, and M. Jansen, Phys. Rev. Lett. 106, 157206 (2011).

[12] I. I. Mazin, D. I. Khomskii, R. Lengsdorf, J. A. Alonso, W. G. Marshall, R. M. Ibberson, A. Podlesnyak, M. J. Martínez-Lope, and M. M. Abd-Elmeguid, Phys. Rev. Lett. 98, 176406 (2007).

[13] G. Jackeli and G. Khaliullin, Phys. Rev. Lett. 101, 216804 (2008).

[14] Y. J. Shin, J. P. Doumerc, P. Dordor, C. Delmas, M. Pouchard, and P. Hagenmuller, J. Solid State Chem. 107, 303 (1993).

[15] A. Wichainchai, P. Dordor, J. P. Doumerc, E. Marquestaut, M. Pouchard, and P. Hagenmuller, J. Solid State Chem. 74, 126 (1988).

[16] T. Sörgel and M. Jansen, Z. Anorg. Allg. Chem. 631, 2970 (2005).

[17] T. Sörgel and M. Jansen, J. Solid State Chem. 180, 8 (2007).

[18] J.-S. Kang, S. S. Lee, G. Kim, H. J. Lee, H. K. Song, Y. J. Shin, S. W. Han, C. Hwang, M. C. Jung, H. J. Shin, B. H. Kim, S. K. Kwon, B. I. Min et al., Phys. Rev. B 76, 195122 (2007).

[19] A. I. Coldea, L. Seabra, A. McCollam, A. Carrington, L. Malone, A. F. Bangura, D. Vignolles, P. G. van Rhee, R. D. McDonald, T. Sörgel, M. Jansen, N. Shannon, and R. Coldea, Phys. Rev. B 90, 020401(R) (2014).

[20] M. Kaneko and M. Ogata, J. Phys. Soc. Jpn. 75, 014710 (2006).

[21] C. Hotta and N. Furukawa, Phys. Rev. B 74, 193107 (2006).

[22] M. Miyazaki, C. Hottam, S. Miyahara, K. Matsuda, and N. Furukawa, J. Phys. Soc. Jpn. 78, 014707 (2009).

[23] L. Cano-Cortés, J. Merino, and S. Fratini, Phys. Rev. Lett. 105, 036405 (2010).

[24] L. Cano-Cortés, A. Ralko, C. Février, J. Merino, and S. Fratini, Phys. Rev. B 84, 155115 (2011).

[25] J. Merino, A. Ralko, and S. Fratini, Phys. Rev. Lett. 111, 126403 (2013).

[26] F. Vernay, K. Penc, P. Fazekas, and F. Mila, Phys. Rev. B 70, 014428 (2004).
[27] J. Kanamori, Prog. Theor. Phys. 30, 275 (1963).

[28] A. Georges, L. de' Medici, and J. Mravlje, Annu. Rev. Condens. Matter Phys. 4, 137 (2013).

[29] H. Uchigaito, M. Udagawa, and Y. Motome, J. Phys. Soc. Jpn. 80, 044705 (2011).

[30] R. Cortés, A. Tejeda, J. Lobo-Checa, C. Didiot, B. Kierren, D. Malterre, J. Merino, F. Flores, E. G. Michel, and A. Mascaraque, Phys. Rev. B 88, 125113 (2013).

[31] Setting $t=0.2 \mathrm{eV}$ to reproduce the bandwidth $W=9 t \sim 2 \mathrm{eV}$ calculated by density-functional theory [17] and evaluating the direct Coulomb repulsion at the Ni-Ni distance $d \simeq 2.9 \AA$ yield $V / t=2-3$, assuming typical values of the dielectric constant $\varepsilon=4-6$ in oxides.

[32] C. Février, S. Fratini, and A. Ralko, arXiv:1503.05714.

[33] L. de' Medici, J. Mravlje, and A. Georges, Phys. Rev. Lett. 107, 256401 (2011).

[34] L. de’ Medici, Phys. Rev. B 83, 205112 (2011).

[35] E. M. Wheeler, R. Coldea, E. Wawrzynska, T. Sorgel, M. Jansen, M. M. Koza, J. Taylor, P. Adroguer, and N. Shannon, Phys. Rev. B 79, 104421 (2009).

[36] It is known that the ordering realized on the triangular lattice with nearest-neighbor antiferromagnetic coupling is a canted antiferromagnet, while the experimentally observed collinear stripe ordering requires a sizable next-nearest-neighbor coupling [10,35,37,46,47], which however, cannot be directly calculated within our method.

[37] L. Seabra, T. Momoi, P. Sindzingre, and N. Shannon, Phys. Rev. B 84, 214418 (2011).

[38] J.-H. Chung, J.-H. Lim, Y. J. Shin, J.-S. Kang, D. Jaiswal-Nagar, and K. H. Kim, Phys. Rev. B 78, 214417 (2008).

[39] J. Merino and R. H. McKenzie, Phys. Rev. B 61, 7996 (2000).

[40] J. Merino, M. Dumm, N. Drichko, M. Dressel, and R. H. McKenzie, Phys. Rev. Lett. 100, 086404 (2008).

[41] X. Deng, A. Sternbach, K. Haule, D. N. Basov, and G. Kotliar, Phys. Rev. Lett. 113, 246404 (2014).

[42] P. Gegenwart, Q. Si, and F. Steglich, Nat. Phys. 4, 186 (2008).

[43] Y. Luo, L. Pourovskii, S. Rowley, Y. Li, C. Feng, A. Georges, J. Dai, G. Cao, Z. Xu, Q. Si, and N. P. Ong, Nat. Mater. 13, 777 (2014).

[44] H. Yoshida, Y. Muraoka, T. Sörgel, M. Jansen, and Z. Hiroi, Phys. Rev. B 73, 020408(R) (2006).

[45] S. A. J. Kimber, M. S. Senn, S. Fratini, H. Wu, A. H. Hill, P. Manuel, J. P. Attfield, D. N. Argyriou, and P. F. Henry, Phys. Rev. Lett. 108, 217205 (2012).

[46] L. Seabra and N. Shannon, Phys. Rev. Lett. 104, 237205 (2010).

[47] Th. Jolicoeur, E. Dagotto, E. Gagliano, and S. Bacci, Phys. Rev. B 42, 4800(R) (1990). 